\title{
Hypoplastic right retro-oesophageal aortic arch: similarities to interrupted aortic arch
}

\author{
W B KNIGHT \\ From the Department of Cardiology, Adelaide Children's Hospital, North Adelaide, South Australia
}

SUMMARY At echocardiography a dysmorphic neonate was found to have a hypoplastic mitral valve, left ventricle, and ascending aorta with interruption of the aortic arch between the left common carotid and the left subclavian arteries-the left subclavian artery arose from a normal sized left descending aorta. Cardiac catheterisation and subsequent necropsy confirmed this arrangement but also showed a tortuous right retro-oesophageal aortic arch. This arch was severely hypoplastic distal to the right subclavian artery. These cardiovascular anomalies occurred in the child of a family in which other members showed the velo-cardio-facial syndrome, a condition known to be associated with right aortic arch. This aortic arch arrangement may be impossible to distinguish from simple aortic interruption by echocardiography alone.

Cross sectional echocardiography lends itself to the detection of interruption of the aortic arch but the following is an unusual case of a rare arch anomaly with similar clinical findings that can be indistinguishable from simple aortic interruption by echocardiography alone.

\section{Case report}

Dysmorphic features were noted soon after birth in a full term $2.8 \mathrm{~kg}$ male infant: prominent nose, short mouth, micrognathia, anomalous ears, widely spaced nipples, arachnodactyly, and minor syndactyly of the second and third toes. A large, tense congenital phimosis required surgical incision. There was no erectile tissue palpable in the penis and the glans was not identifiable. He was in mild heart failure with no evidence of cyanosis of upper or lower body. The only palpable peripheral pulse was the right brachial and this was weak. Systolic blood pressures were $70 \mathrm{~mm} \mathrm{Hg}$ in the right arm, $67 \mathrm{~mm} \mathrm{Hg}$ in the left arm, and $85 \mathrm{~mm} \mathrm{Hg}$ in the legs. There was a prominent parasternal impulse. The second heart sound was narrowly split with an accentuated pulmonary component. There was an ejection click and a soft basal ejection systolic murmur. There was no stridor. The electrocardiogram showed right ventricular hyper-

Requests for reprints to Dr W B Knight, Department of Cardiology, Adelaide Children's Hospital, 72 King William Road, North Adelaide, South Australia 5006, Australia. trophy and the chest radiograph showed mild cardiomegaly and pulmonary congestion. The thymus obscured details of the aortic arch and there was no obvious tracheal deviation.

Cross sectional echocardiography showed normal atrioventricular and ventriculoarterial connections. The right heart chambers were enlarged. The left atrium, mitral valve, and left ventricle were hypoplastic. The end diastolic left ventricular dimension was only $10 \mathrm{~mm}$. A perimembranous ventricular septal defect of moderate size was seen. The pulmonary artery was much larger than the ascending aorta, which had a diameter of only $6 \mathrm{~mm}$. The aortic valve was small but otherwise normal. The ascending aorta did not arch posteriorly but simply bifurcated. The descending aorta was to the left of the spine and of normal size. It was connected to the pulmonary artery by way of the ductus arteriosus, which was only slightly constricted. The left subclavian artery arose from its postero-superior extremity.

Cardiac catheterisation showed a large left to right atrial and ventricular shunt with equal systolic pressures in both ventricles and pulmonary trunk. There was a $20 \mathrm{~mm} \mathrm{Hg}$ systolic pressure drop across the ductus arteriosus to the descending aorta. The ascending aorta was not entered. Cineangiography confirmed the echocardiographic details of cardiac anatomy. Separate injections of contrast into the left ventricle (fig la and b) and ductus arteriosus (fig 1c) confirmed a lack of continuity between the left common carotid and left subclavian arteries. However, the right branch of the bifurcation of the ascending aorta was not an innominate artery-as 

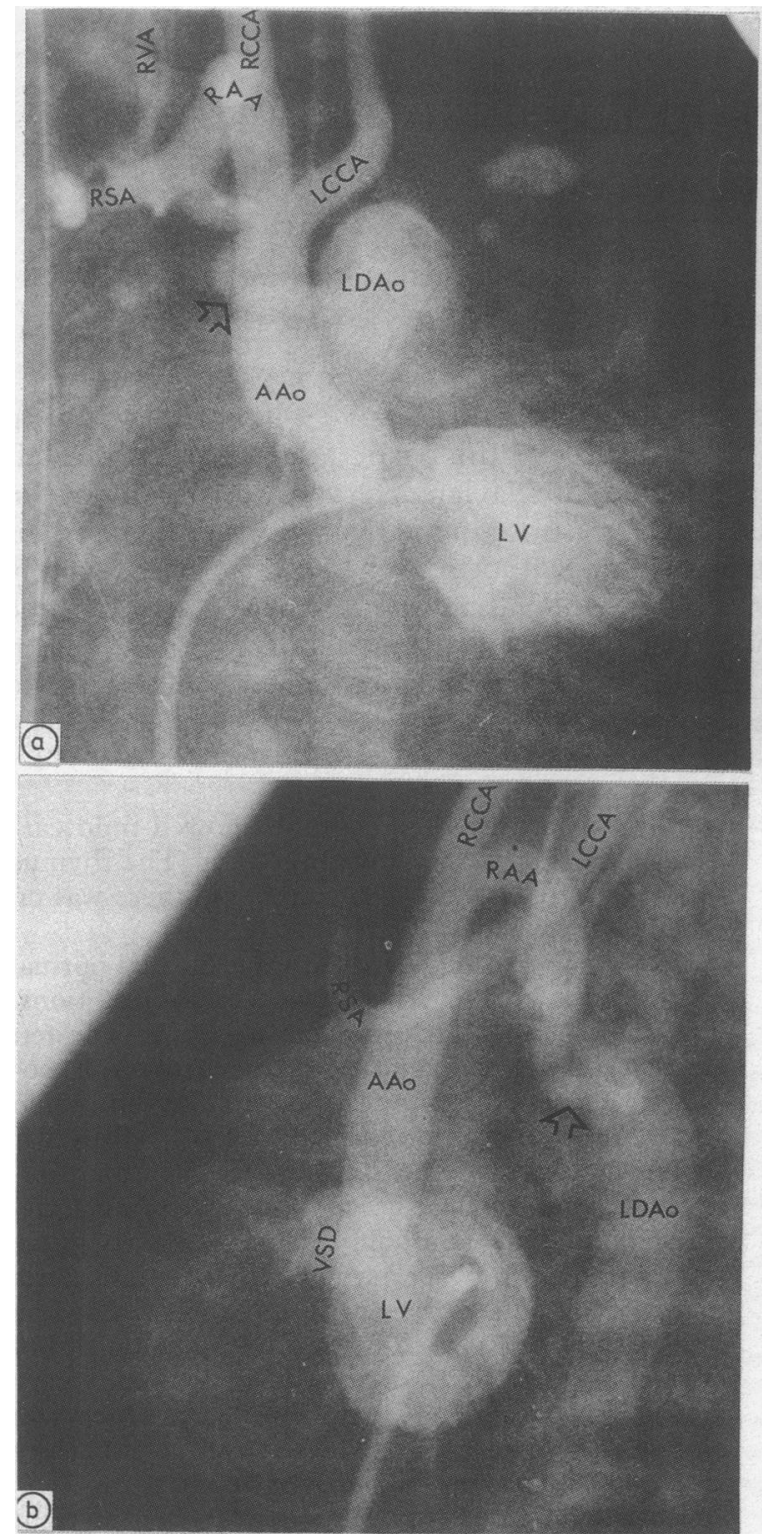

had been assumed from echocardiography. It gave rise to the right common carotid artery and then arched posteriorly to the right of the trachea to give rise to the right subclavian artery. Beyond this point it became narrow (minimal internal diameter $2 \mathrm{~mm}$ ) and tortuous and crossed the midline behind the oesophagus to join the left descending aorta just distal to the origin of the latter from the partly constricted arterial duct (fig 1c). It was thus a retrooesophageal right aortic arch, severely hypoplastic

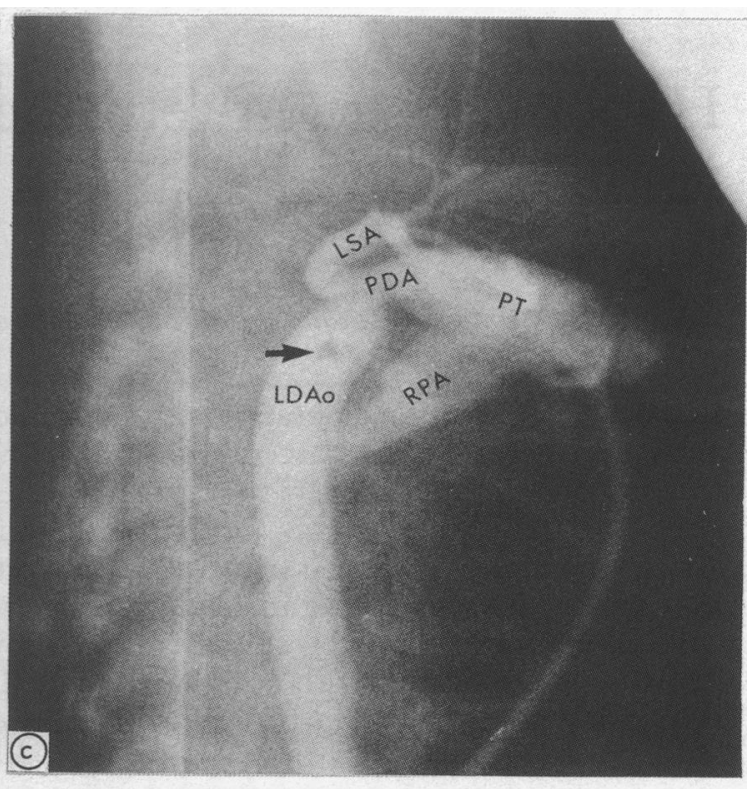

Fig 1 Cineangiograms showing left ventriculogram in (a) $30^{\circ}$ right and (b) $60^{\circ}$ left anterior oblique projections. $L V$, left ventricle, $A A$ o, ascending aorta, $R A A$, right aortic arch, $R C C A, L C C A$, right and left common carotid arteries;

$R S A, R V A$, right subclavian and vertebral arteries; $L D A o$, left sided descending aorta. The open arrows point to the tortuous, hypoplastic distal retro-oesophageal right aortic arch. (c) Lateral view of injection into the partly constricted ductus arteriosus (PDA).PT, pulmonary trunk, $R P A$, right pulmonary artery. Solid arrow points to the wash-in of uncontrasted blood where the distal right aortic arch joins the left sided descending aorta.

and tortuous in its distal portion, associated with an anomalous origin of the left subclavian artery.

Serum calcium, $T$ cell function, and numbers and chromosome analysis were normal. He died when he was nine days old. Necropsy confirmed the cardiovascular abnormalities including small tortuous right aortic arch (figs $2 a$ and $b$ ). The ventricular septal defect accepted a probe of $6 \mathrm{~mm}$. The ascending aorta was only $4 \mathrm{~mm}$ in diameter. The aortic valve was small but not stenotic. A persistent left superior 

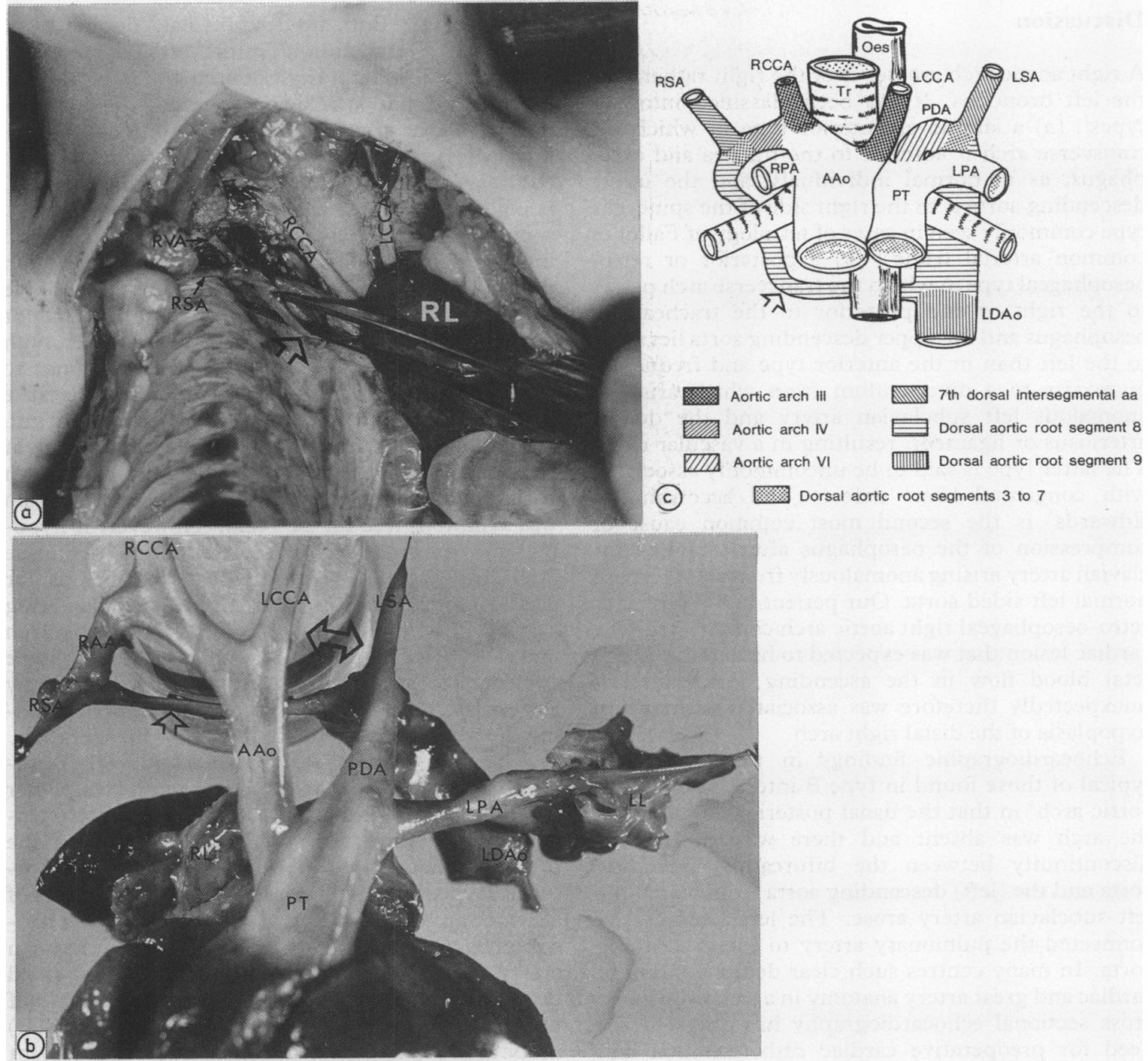

Fig 2 (a) Anatomical specimen showing right aortic arch looped around forceps, passing behind the oesophagus and (b) the dissected heart and great arteries as seen from the front. The double headed arrow shows the "interruption" between the left common carotid artery and left subclavian artery while the open arrow points to the stretched hypoplastic distal right aortic arch. $L P A$, left pulmonary artery; $L L, R L$, hilar portions of left and right lungs. See legend to fig 1 for other abbreviations. (c) Diagram of $(b)$ indicating the presumed embryological origin of the vascular structures. Tr, trachea, Oes, oesophagus.

vena cava drained to the coronary sinus. Only a very small amount of penile erectile tissue was present. The remainder of the renal tract was normal. An unsuspected finding was a bifid uvula.

The father of this patient has a left aortic arch and no known cardiac abnormalities, but he has dysmorphic facial features including narrow palpebral fissures, malar and maxillary hypoplasia, a prominent nose with tented alae nasi, short philtrum, large mouth, and bat ears. He has a repaired cleft palate. He described himself as a slow learner. A subsequent son was born with similar facial dysmorphism and bifid uvula. He has undergone open heart surgery for a large ventricular septal defect. The father and two sons are thought to have the velo-cardio-facial syndrome. 


\section{Discussion}

A right aortic arch crosses over the right rather than the left bronchus. It has been classified into two types $^{1}$ : (a) a simple or anterior type in which the transverse arch is anterior to the trachea and oesophagus, as in normal individuals, and the upper descending aorta is to the right side of the spine (the type commonly seen in cases of tetralogy of Fallot or common arterial trunk); (b) a posterior or retrooesophageal type in which the transverse arch passes to the right of and posterior to the trachea and oesophagus and the upper descending aorta lies more to the left than in the anterior type and frequently gives rise to a diverticulum from which arise an anomalous left subclavian artery and the ductus arteriosus or ligament, resulting in a vascular ring. ${ }^{2}$ The latter type is said to be uncommonly associated with congenital heart defects ${ }^{1}$ and according to Edwards ${ }^{3}$ is the second most common cause of compression of the oesophagus after a right subclavian artery arising anomalously from an otherwise normal left sided aorta. Our patient had a posterior retro-oesophageal right aortic arch complicated by a cardiac lesion that was expected to have reduced the fetal blood flow in the ascending aorta and not unexpectedly therefore was associated with severe hypoplasia of the distal right arch.

Echocardiographic findings in this case were typical of those found in type B interruption of the aortic $\operatorname{arch}^{4}$ in that the usual posterior curvature of the arch was absent and there was an obvious discontinuity between the bifurcating ascending aorta and the (left) descending aorta from which the left subclavian artery arose. The left arterial duct connected the pulmonary artery to the descending aorta. In many centres such clear demonstration of cardiac and great artery anatomy in a sick neonate by cross sectional echocardiography has obviated the need for preoperative cardiac catheterisation and cineangiography. Accurate echocardiographic diagnosis of the abnormality of the aortic arch in our patient was made difficult by the hypoplasia and tortuosity of the distal right arch. In addition, because the echocardiographic appearances were consistent with the clinical signs, the presence of an obstructive right retro-oesophageal aortic arch was neither suspected nor specifically searched for. To my knowledge there have been only two previous descriptions of the echocardiographic appearances of retro-oesophageal right aortic $\operatorname{arch}^{56}$ - both had a right cervical aortic arch.

In a search of published reports I found two cases of retro-oesophageal right aortic arch with an aberrant left subclavian artery and hypoplastic distal right arch, both associated with a large ventricular septal defect. ${ }^{7^{8}}$ Both are described as "double aortic. arch" with "interruption" of the left arch, despite $\overrightarrow{\text { " }}$ " stated lack of continuity between the left commonf carotid and left subclavian arteries. In so far as normal aortic arch anatomy, with one continuous arch (the left one) and persisting remnants of the contralateral arch (such as the innominate artery being derived from the right fourth aortic arch), could also be described as double aortic arch with $\overrightarrow{0}$ interruption of one of the arches, this descriptiondoes not seem useful or logical, particularly if double్లు aortic arch is defined by the presence of luminal or fibrous continuity of bilateral aortic arches. In both the reported cases ${ }^{78}$ there was a good response to pulmonary banding alone, presumably because? hypoplasia of the right aortic arch was not critical $\checkmark$ Indeed left aortic arch atresia with hypoplastic righto retro-oesophageal arch (that is double aortic arch. with aortic arch atresia) was reported in a patient who was symptom free for 21 years. ${ }^{9}$ However, if hypo $\frac{\mathbb{}}{8}$ plasia of the right arch is severe enough, definitive arch surgery may be required as urgently as fo: classic aortic interruption. The vascular ring associated with right retro-oesophageal aortic archo and left arterial duct does not usually produceso compressive symptoms in infancy, but these mayo appear later in life. ${ }^{10}$ Hence the importance of divid ing the ring at the time of definitive aortic operation.

It has been noted that in patients with right retro oesophageal arch, the arch often extends to a highe $\frac{1}{2}$ level in the thorax than in patients without a retro $-\frac{}{\vec{T}}$ oesophageal segment. ${ }^{10}$ This can be seen in the present patient (fig 2a). In addition, obvious tor $-\frac{5}{-}$ tuosity is seen on angiography (fig 1a). Some cases off cervical arch, most of which have a right retrooesophageal segment and anomalous left subclaviand artery arising from a diverticulum on the left sided descending aorta, may represent the extreme end of the spectrum of retro-oesophageal right (or left ${ }^{2}$. aortic arch in which there is considerable impairmen? of the normal contraction of the aortic arch and consequent failure of its migration into the chest.

There seems little doubt that this patient, his brother, and father have the velo-cardio-facial syn drome, ${ }^{11}{ }^{12}$ an inherited condition comprising cleff palate (either overt or submucous), learning dis ${ }_{\pi}$ ability, facial dysmorphism, and cardiovascular malo formations. Although thought to be autosoma dominant, male to male transmission has not beent described until recently. ${ }^{13}$ The mode of inheritance iff this family seems to confirm the autosomal dominane nature of this condition. In a survey of 39 patients with velo-cardio-facial syndrome, cardiovascular anomalies occurred in $84 \% .{ }^{12}$ The most commors malformations were ventricular septal defect and right aortic arch, the latter being of both anterior and 
posterior types. ${ }^{14}$ These were both present in the index case as well as persistent left superior vena cava, another known associated anomaly. A subsequent sibling had a large ventricular septal defect requiring surgical closure at three weeks of age. To my knowledge, hypoplasia of the left heart structures has not been reported to occur in velo-cardio-facial syndrome. Similarly, the penile anomaly is not a recognised feature. Fetal echocardiography may have an important role in the prenatal detection of this syndrome.

\section{References}

1 Eisen D. Right aortic arch with report of eight cases. Radiology 1944;42:570-8.

2 Gross RE, Ware PF. The surgical significance of aortic arch anomalies. Surg Gynecol Obstet 1946;83:435-48.

3 Edwards JE. Retro-oesophageal segment of the aortic arch, right ligamentum arteriosum causing congenital vascular ring about the trachea and esophagus. Mayo Clin Proc 1948;23:108-16.

4 Celoria GC, Patton RB. Congenital absence of the aortic arch. Am Heart J 1959;58:407-14.

5 D'Cruz IA, Stanley A, Vitullo D, Desai P, Chiemmongkoltip P. Non-invasive diagnosis of right cervical aortic arch. Chest 1983;83:820-2.

6 Kveselis DA, Snider AR, Dick M, Rocchini AP. Echocardiographic diagnosis of right aortic arch with a retroesophageal segment and left descending aorta. Am J Cardiol 1986;57:1198-9.

7 Brenner JI, Berman MA, Talner NS, Stansel HC. Double aortic arch system producing obstruction to left ventricular outflow. Am J Cardiol 1974;34:854-6.

8 Collins-Nakai RL, Parisi-Buckley L, Fyler DC, Castaneda AR. Interrupted aortic arch in infancy. $J$ Pediatr 1976;88:959-62.

9 Blackford LM. Coarctation of the aorta. Arch Intern Med 1928;41:702-35.

10 Felson B, Palayew MJ. The two types of right aortic arch. Radiology 1963;81:745-59.

11 Strong WB. Familial syndrome of right-sided aortic arch, mental deficiency, and facial dysmorphism. $J$ Pediatr 1968;73:882-8.

12 Shprintzen RJ, Goldberg RB, Young D, Wolford L. The velo-cardio-facial syndrome: a clinical and genetic analysis. Pediatrics 1981;67:167-72.

13 Williams MA, Shprintzen RJ, Goldberg RB. Male-tomale transmission of the velo-cardio-facial syndrome: a case report and review of 60 cases. J Craniofac Genet Dev Biol 1985;5:175-80.

14 Young D, Shprintzen RJ, Goldberg RB. Cardiac malformations in the velocardiofacial syndrome. Am J Cardiol 1980;46:643-8. 